\title{
The Design of An Aquaculture Environmental Monitoring System Based on Zigbee
}

\author{
Ping-ping Xiao ${ }^{1,}$, , Chao Wang ${ }^{1}$ \\ ${ }^{1}$ School of Electrical Informationline, Changchun Guanghua University, China \\ a286444752@qq.com
}

\section{Keywords: Aquaculture, ARM11, ETC6410, embedded ZigBee}

Abstract. For solving the current situation of monitoring and management in traditional aquaculture farms, the design idea of aquaculture environmental monitoring system was put forward with the development of intelligence and the maturity of intelligent technology in recent years. We described in detail the whole process of design and development of aquaculture environmental monitoring System Based on ARM11 architecture in the paper. After the comprehensive demonstration of the design plan, we focused on the research of ZigBee wireless communication technology, serial communication technology and network communication technology.

\section{Introduction}

Aquatic products (including aquatic plants and aquatic animals) are indispensable in human life. But with the expansion of human activities, the natural growth of aquatic products had been greatly affected. In order to protect the nature, the government required that the annual ban began more and more time in advance, and the duration time was getting longer, so the demands of artificial aquaculture products were increasing, thus the urgent demand was to reduce the cost of farming for aquaculture field.

Aquaculture has a strict requirements on water conditions, such as water quality, light intensity, water temperature and water level. The traditional aquaculture field adopted the artificial mode to monitor and control, it not only spent a lot of manpower, but also often accompanied by a large error. Therefore, the design of this system is to solve these problems, in order to achieve the real-time monitoring, automatic adjust the growth conditions of aquatic products, reduce labor costs and improve the accuracy.

Considering the shortcomings of the general monitoring system, such as the difficulty of underwater wiring, the difficulty of maintenance and the high cost, it is proposed that the advanced ZigBee wireless technology will be used to achieve remote wireless monitoring.

\section{Realization Function}

The goal of this system was to design an online monitoring and control system for water quality in aquaculture field, which could realize real-time acquisition, transmission, processing and feedback of multiple environmental parameters. The system took water quality parameters such as water temperature, nutrient, $\mathrm{pH}$ value and pond level as monitoring objects. The main function of the system covered data collection, transmission, query and data processing, and realized real-time monitoring and environmental control of the data. The design system adopted a layered and distributed system structure. The system consisted of the following three layers:

1. The lowest level was data acquisition and wireless data interaction through ZigBee wireless communication technology and network coordinator, and collected data to obtain various environmental parameters through distributed structure. We put the multiple data acquisition nodes in different locations of aquaculture ponds, and each data acquisition node could mount multiple sensors at the same time to achieve data acquisition for multiple environmental parameters. The data acquisition nodes were realized the ad-hoc network by ZigBee network technology, then the location 
information and the collected data were transmitted to the coordinator module by the ZigBee wireless communication technology.

2. The middle layer was a data aggregation layer consisted of an embedded ARM processor and an embedded Web server. The coordinator node received the data collected by all terminal nodes under the network and transferred the data to the ARM processor through the communication protocol. The processor handled the received data, and was also responsible for building a Web server and provided a human-computer interface. This part was extended on the basis of embedded micro controller 6410 chip, and the embedded Linux was transplanted on it, which was developed according to the requirements.

3. The top layer was PC and mobile terminals. PC could realize real-time data monitoring and system management through a network browser or a host computer program. The mobile phone could monitor and manage the data in real time through the mobile browser of the common network.

\section{The Scheme Design}

In order to achieve real-time monitoring of multi-point data in a wide range, the system implemented the online monitoring framework of three layers of host computer interaction interface, consisted of ZigBee data collection module, forwarding module, and data processing module.

The three layer architecture of the system was shown in Figure 1.

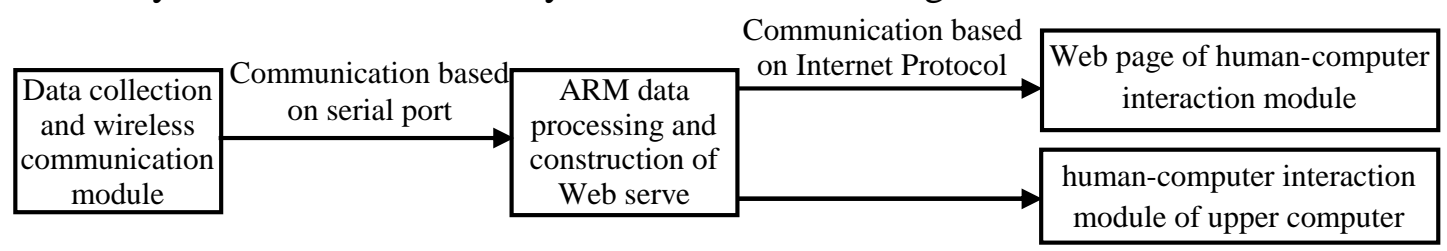

Fig. 1 The system architecture

We built the monitoring system based on the wireless sensor network. The system had the function of self organising dynamic star network by collecting data from the bottom and sending data to the processor to provide terminal human-computer interface. It could complete the task of monitoring water quality, and the number of nodes was easy to expand. The overall structure of the system was shown in Figure 2.

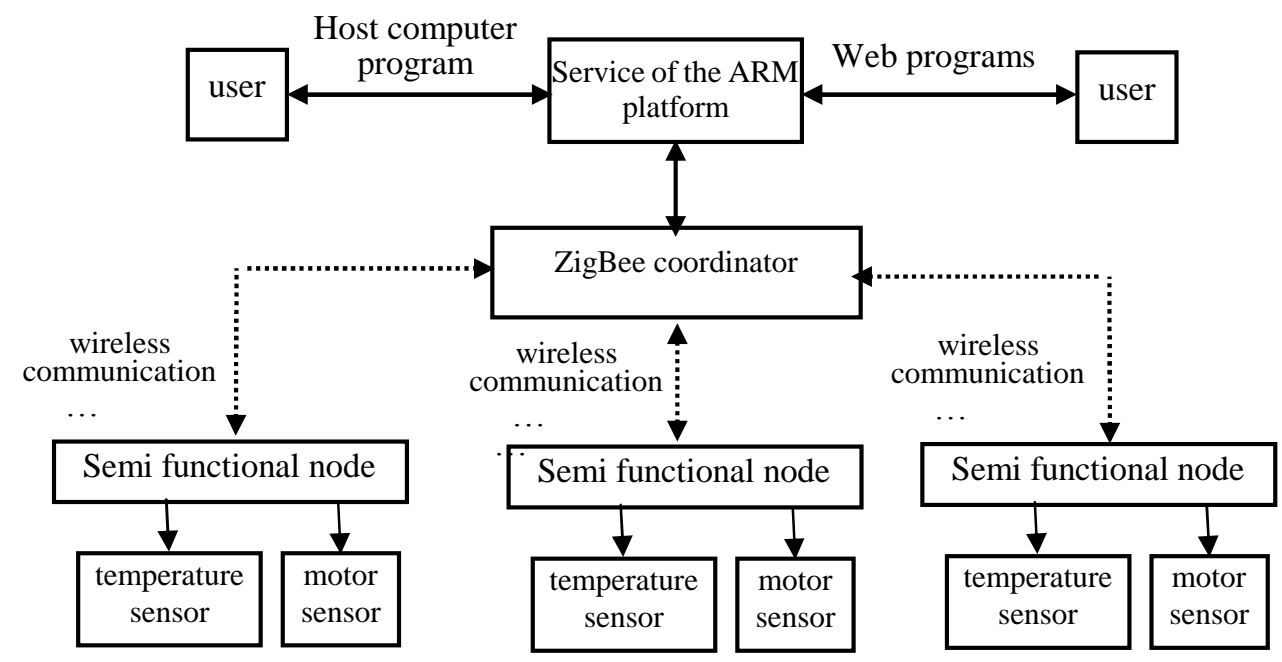

Fig. 2 The overall structure of the system

\section{The Hardware Design}

S3C6410 is a 16/32 bit RISC microprocessor based on ARM11 architecture, which provides a cost-effective application processor and low power consumption and high performance solutions for 
the embedded market. It integrated many powerful hardware unit, and provided the abundant internal equipment for the user, thereby reduced the total cost of the system and improved the overall function.

In the transmission of the collected data, the system mainly used the ZigBee infinite radio chip $\mathrm{CC} 2530$ as the half function module of the microprocessor and the radio transceiver. The photosensitive sensor module used the partial pressure of a photosensitive resistor, and then converted the analog signal into a digital signal to achieve the data acquisition of light intensity. The temperature sensor was mounted on the IIC bus to collect the temperature. The function of QM-NG1 gas sensor used a simple circuit to convert the change of the electrical conductivity to an output signal corresponding to the concentration of the gas. When the carbon dioxide gas existed in the environment of the sensor, the conductivity of the sensor increased with the increase of gas concentration. Motor driving circuit LG9110 driver was a two channels push-pull power amplifier application for specific integrated. The two output terminal could directly drive the motor forward and reverse movement, it had high current driving ability for controlling and driving motor.

\section{System Test}

According to the function of each module, the system was divided into five parts: water level monitoring, nourishment injection, lighting monitoring, temperature monitoring and water quality monitoring. After the connection between host computer and server was successful, we recorded the test results sequentially.

The results of water level monitoring module. The hardware sensor nodes used ultrasonic sensor nodes and motor sensor nodes in this module, and the software control interface was the interface of water exchange system. The test effect was shown in Figure 3. When you clicked the injection button, the stepper motor of the motor sensor nodes rotated clockwise. When you clicked the stop button, the stepper motor of the motor sensor nodes stopped rotating. When you clicked the drainage button, the stepper motor of the motor sensor nodes rotated counterclockwise.

The results of feed injection module. The hardware sensor nodes used in this module had the motor sensor nodes, and the software control interface used is the interface of feed injection system. Every injection time was default to 60 seconds, it stopped automatically, and it can be stopped manually. The test effect was shown in Figure 4.When you clicked the injection button, the red light of motor sensor nodes was lighted, when you clicked the stop button, the light was off.

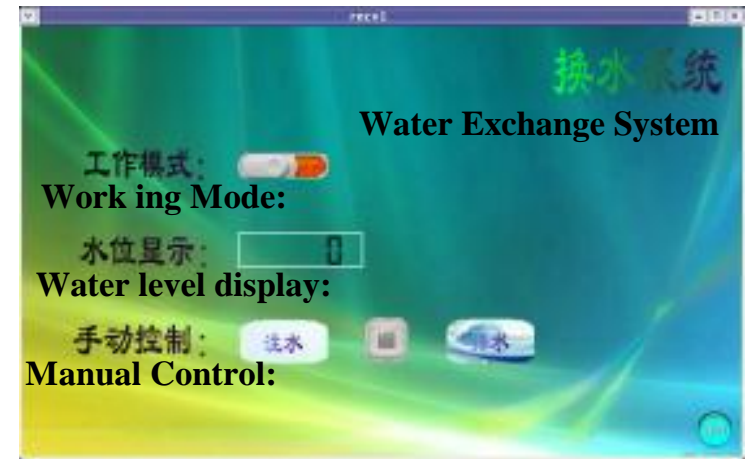

Fig. 3 The result of water level module

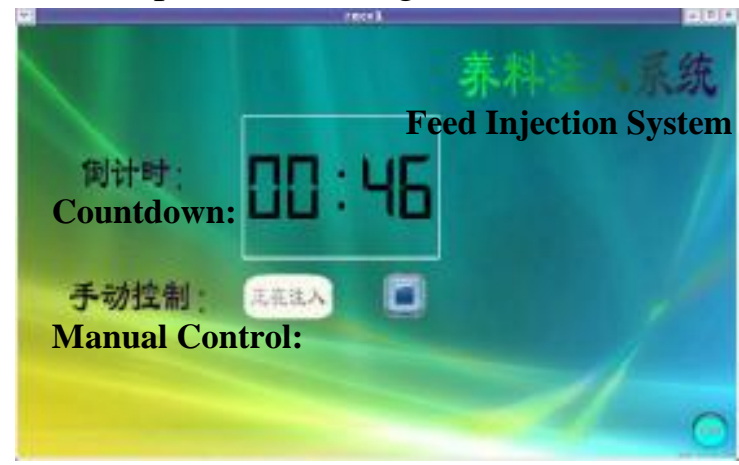

Fig. 4 The result of water level module

The results of light monitoring module. The hardware sensor nodes used in this module had photosensitive sensor node and motor sensor node, the work mode was default to automatic control mode. The light intensity monitoring part displayed the data returned by the photosensitive sensor node in real time. Under manual control, the control button of the shading curtain was non-clickable state. When the working mode was switched to manual mode, the shutter control button changed to clickable state, and the illumination intensity monitoring result was still displayed in real time. The test effect was shown in Figure 5.

The results of temperature monitoring module. The hardware sensor nodes used in this module included temperature sensor nodes and LED dimming lamp sensor nodes. The software test effect was shown in Figure 5. Under the manual work mode, when the button was clicked " + " number button, 
the temperature of the air conditioning rised, the light on the LED sensor node became brighter and the temperature of the air conditioner rised. When the click "-" button was clicked, the temperature of the air conditioner drop, the light on the sensor node gradually darkened, representing a drop in the temperature of the air conditioner.
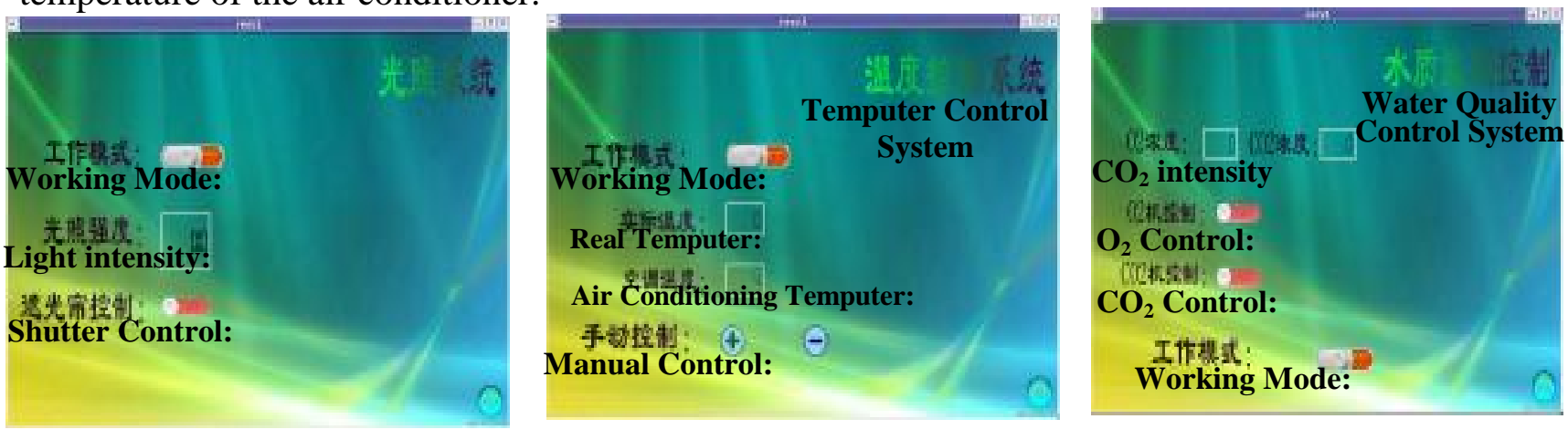

Fig. 5 The result of the light module Fig. 6 The results of temperature module Fig. 7 The results of water quality module

The result of water quality monitoring module. The hardware sensor nodes used in this module included temperature sensor node, gas sensor node and motor sensor node. Because of hardware limitation, humidity is used to replace oxygen concentration. The test effect was shown in Figure 7. When the $\mathrm{O} 2$ control button switched to the "no" state, the white light of motor sensor node was lighted, $\mathrm{O} 2$ machine opened, began to increase the oxygen concentration in the water; when switched to the "off" state, the light put out, $\mathrm{O} 2$ machine closed. When the $\mathrm{CO} 2$ control button switched to the "no" state, the green light of motor sensor nodes was lighted, $\mathrm{CO} 2$ machine open, began to increase in water gas concentration; when switched to the "off" state, the lights burn out, CO2 machine closed.

\section{Conclusion}

This design integrated serial communication technology, ZigBee wireless communication technology and network communication technology, and developed a complete set of aquaculture environmental monitoring system system, highlighted the characteristics of intelligent embedded devices. It improved the reliability, extensibility and portability of the system, and the whole system had high stability and high transplantability.

\section{Acknowledgements}

This work was financially supported by the Jilin province industrial technology research and development of special projects.

\section{References}

[1] Robust adaptive neuro-fuzzy control of uncertain nonholonomic systems[J]. Shuzhi Sam GE,Chee Khiang PANG,Tong Heng LEE. Journal of Control Theory and Applications. 2010(02).

[2] A review of wire-less sensors and networks' 'applications in agriculture. Rehman A,Abbasi A Z,Islam N,et al. Computers and Standards . 2011.

[3] Wireless sensors in agriculture and food industry-Recent development and future perspective. Wang N,Zhang N,Wang M. Computers and Electronics in Agriculture . 2006.

[4] Development of wireless sensornetwork for combustible gas monitoring. Andrey Somova,Alexander Baranov,Alexey Savkin et al. Sensors and Actuators . 2011. 
[5] Energy-efficient link layer for wirelessmicrosensor networks. E Shih,B H Calhoun,S Cho,et al. IEEE Computer Society Workshop on VLSI 2001 . 2001. 